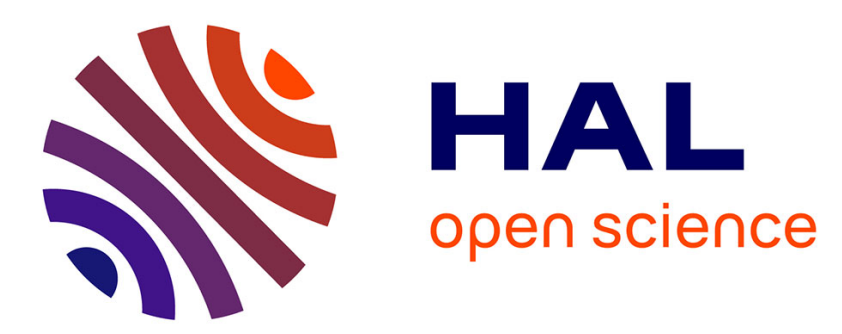

\title{
Effect of normal compression on the shear modulus of soft tissue in rheological measurements
}

Mehmet Ayyildiz, Soner Cinoglu, Cagatay Basdogan

\section{To cite this version:}

Mehmet Ayyildiz, Soner Cinoglu, Cagatay Basdogan. Effect of normal compression on the shear modulus of soft tissue in rheological measurements. Journal of the mechanical behavior of biomedical materials, 2015, 49, pp.235-243. 10.1016/j.jmbbm.2015.05.011 . hal-03177310

\section{HAL Id: hal-03177310 \\ https://hal.science/hal-03177310}

Submitted on 23 Mar 2021

HAL is a multi-disciplinary open access archive for the deposit and dissemination of scientific research documents, whether they are published or not. The documents may come from teaching and research institutions in France or abroad, or from public or private research centers.
L'archive ouverte pluridisciplinaire HAL, est destinée au dépôt et à la diffusion de documents scientifiques de niveau recherche, publiés ou non, émanant des établissements d'enseignement et de recherche français ou étrangers, des laboratoires publics ou privés. 


\title{
Effect of Normal Compression on the Shear Modulus of Soft Tissue in Rheological Measurements
}

\author{
Mehmet Ayyildiz, Soner Cinoglu, Cagatay Basdogan* \\ College of Engineering, Koc University, Istanbul \\ *Corresponding author: Email address: cbasdogan@ku.edu.tr. \\ Address: College of Engineering, Koc University, Istanbul, 34450, Turkey. \\ Tel.: +90 212338 1721; Fax: +90212338 1548
}

\begin{abstract}
In experiments performed to characterize rheological properties of viscoelastic solids, some amount of compression is necessary to prevent the slippage between the sample and the parallel plates of a rheometer. While the effect of normal compression on the shear rheological properties of viscoelastic materials has been already acknowledged and reported in few studies in the literature, to our knowledge, no systematic study has been conducted to investigate this effect in detail to date. In this study, we perform two sets of experiments to investigate the effect of normal strain and strain rate on the dynamic shear moduli of bovine liver. First, we apply normal compressive strain to the cylindrical bovine samples up to $20 \%$ at loading rates of $v=0.000625,0.00625,0.0625$, 0.315, $0.625 \mathrm{~mm} / \mathrm{s}$. Second, we perform torsional shear loading experiments, in the frequency range of $\omega=0.1$ to $10 \mathrm{~Hz}$, under varying amounts of compressive pre-strain $(\varepsilon=1 \%, 2.5 \%, 5 \%, 7.5 \%, 10 \%, 12.5 \%, 15 \%, 17.5 \%$ and $20 \%)$ applied at the quasi-static loading rate of $\mathrm{v}=0.000625 \mathrm{~mm} / \mathrm{s}$. The results of the experiments show that the shear moduli of bovine liver increase with compressive pre-strain. A hyper-viscoelastic constitutive model is developed and fit to the experimental data to estimate the true shear moduli of bovine liver for zero pre-compression. With respect to this reference value, the mean relative error in the measurement of shear moduli of bovine liver varies between $0.2 \%$ and $243.1 \%$ for the compressive pre-strain varying from $\varepsilon=1 \%$ to $20 \%$.
\end{abstract}


The dynamic shear modulus of bovine liver for compressive pre-strain values higher than $\varepsilon>2.5 \%$ are found to be statistically different than the true shear moduli estimated for zero compressive strain $(\mathrm{p}<0.05)$.

Keywords: Liver, rheology, pre-compression, pre-strain, hyper-viscoelastic, constitutive model, dynamic shear modulus

\section{Introduction}

One of the most frequently used experimental methods for characterizing the viscoelastic material properties of soft tissue is the dynamic oscillation experiment. In this test, small periodic strains at varying frequencies are applied to the sample and the stress response is recorded. These small-amplitude oscillatory tests are commonly performed in shear using a rheometer. In rheological shear measurements, a cylindrical viscoelastic sample is placed between the plates of a rheometer, and some amount of compression is applied before the oscillations to ensure full contact between the sample and the plates and hence to reduce the slippage. Subsequently, small oscillatory shear is applied to the sample in the linear viscoelastic range (LVR) to measure its torque response. The shear stress is then calculated from the measured torque response based on the sample geometry. Finally, the shear stress is divided by the shear strain in frequency domain to obtain two shear moduli at each frequency: one in-phase with the applied strain, called shear storage modulus, and the other 90 degrees out-of-phase with the applied strain, called shear loss modulus.

Using a rheometer, the frequency-dependent shear material properties of liver (Ayyildiz et al., 2014; Kiss et al., 2004; Klatt et al., 2010; Liu and Bilston, 2000; Tan et al., 2013; Wex et al., 2013), brain (Garo et al., 2007; Hrapko et al., 2008), and kidney (Nasseri et al., 2002; Nicolle and Palierne, 2010) have been measured. Although pre-compression has been applied to the samples in all these studies, its effect on the shear material properties of the soft tissue being tested has been reported in few studies only. Hrapko et al. (2008) showed that increasing the normal force applied on the brain tissue samples results in overestimation of shear modulus. Tan et al. (2013) showed that the magnitude of pre-compression alters the storage shear modulus and LVR of 
bovine liver and recommend not applying more than $10 \%$ pre-strain in the normal direction. Despite these observations, to our knowledge, no systematic study has been conducted to investigate this effect in detail to date. In this study, we perform two sets of characterization experiments (normal compression and dynamic shear loading) to examine the effect of compression on the dynamic shear moduli of bovine liver. A hyper-viscoelastic constitutive model is developed and fit to the experimental data to estimate the dynamic shear moduli of bovine liver at zero pre-strain and compare with the measurements performed at higher prestrains. The constitutive models developed so far for material characterization of liver tissue have targeted to model either its linear (Ayyildiz et al., 2014; Klatt et al., 2010; Liu and Bilston, 2000; Yarpuzlu et al., 2014) or nonlinear (Nasseri et al., 2002; Nicolle et al., 2010) dynamic viscoelastic response in shear loading only, but not under the combined loading of compression and shear.

\section{Materials and Methods}

\subsection{Preparation of Tissue Samples}

In order to obtain cylindrical liver samples, fresh bovine livers are harvested from 3 animals in a local slaughterhouse. The livers are transferred from the slaughterhouse to our laboratory while cold preserved in the Lactated Ringer's solution at $+4^{\circ} \mathrm{C}$. Cylindrical tissue samples with a diameter of $25 \mathrm{~mm}$ and a thickness of $2.5 \pm 0.5 \mathrm{~mm}$ are obtained from each liver using the tissue slicing and sampling apparatus developed in our earlier study (Ayyildiz et al., 2014). A total of 28 tissue samples are obtained from each liver. The samples are kept in sterile specimen cups containing Lactated Ringer's solution until the time of testing. Each sample is tested within 30 minutes to ensure that its material properties are not affected significantly from dehydration and all experiments for each liver are completed within 12 hours after harvesting. Hence, the rim of each sample is covered with the preservation solution during the experiments and it is not exposed to air until the solution evaporates (Nicolle and Palierne, 2010). 


\subsection{Experimentation}

The tissue samples are tested by a shear strain-controlled, parallel plate rheometer (Anton Paar MCR 102, see Fig. 1). In addition to a torque sensor for shear measurements, our rheometer is also equipped with a force sensor, having a range of $0.01 \mathrm{~N}$ to $50 \mathrm{~N}$ with a resolution of 0.01 $\mathrm{N}$, for measuring forces in the normal direction. In order to prevent slippage between the sample and the plates, sand paper is applied to the upper and lower plates of the rheometer. The initial height of each sample is determined by moving the upper plate with a very low velocity of $0.000625 \mathrm{~mm} / \mathrm{s}$ towards to the sample until a contact force of $0.1 \mathrm{~N}$ is reached. Then, the movement of the upper plate is stopped and the gap between the upper and lower plates is measured and taken as the initial height of the sample. Hence, in addition to the use of sandpapers, an initial normal force is applied to each sample during the measurement of its height, which makes the sample surface more flat and reduces the contact problem during the actual characterization experiments. During all measurements, temperature is kept at $24^{\circ} \mathrm{C}$ by the Peltier module (P-PTD200/56/AIR) of the rheometer.

In order to investigate the effect of pre-compression on the dynamic shear response of bovine liver, normal compression and dynamic shear loading experiments are performed on the samples (see Table 1).

\subsubsection{Compression Experiments}

The samples are compressed in normal direction up to $20 \%$ strain. Each sample is compressed only once at a loading rate of $\mathrm{v}=0.000625,0.00625,0.0625,0.315,0.625 \mathrm{~mm} / \mathrm{s}$ and the force response is recorded as a function of displacement. The compression experiments are repeated 4 times at each loading rate with a different sample (see Table 1). Hence, a total of 60 tissue samples are examined in compression $(4$ samples per loading rate $\times 5$ loading rates per liver $\times 3$ livers).

\subsubsection{Dynamic Shear Loading (DSL) Experiments}

In order to determine the LVR of the samples, amplitude sweep experiments are performed under normal compressive strains of $\varepsilon=5 \%, 10 \%, 15 \%$ and $20 \%$, with four samples 
taken from each liver ( 4 samples per liver $\times 3$ livers $=12$ samples). The samples are oscillated in torsion at a constant frequency of $\omega=10 \mathrm{~Hz}$ while the shear strain amplitude is varied from $\gamma=$ $0.1 \%$ to $5 \%$. It is observed that LVR of the bovine liver is decreased with increasing pre-strain, as reported in Tan et al. (2013). The shear strain value corresponding to lowest LVR, $\gamma_{\mathrm{LVR}}=0.5 \%$, is used in all frequency sweep experiments, which are performed in the frequency range of $\omega=$ $0.1 \mathrm{~Hz}$ to $10 \mathrm{~Hz}$ under varying amounts of compressive pre-strain ( $\varepsilon=1 \%, 2.5 \%, 5 \%, 7.5 \%$, $10 \%, 12.5 \%, 15 \%, 17.5 \%$ and $20 \%$ ) applied at the quasi-static loading rate of $\mathrm{v}=0.000625 \mathrm{~mm} / \mathrm{s}$ (see Table 1). The frequency sweep experiments are repeated with four samples taken from each liver ( 4 samples per liver $\times 3$ livers $=12$ samples). In all experiments, the torque response is recorded as a function of frequency.

\subsection{Constitutive Model}

A hyper-viscoelastic constitutive model is developed to investigate the material properties of uniaxially compressed cylindrical liver tissue under torsional shear loading. It utilizes a single parameter Neo-Hookean material model to characterize the quasi-static hyperelastic behavior of liver tissue under large deformations and a twelve-parameter viscoelastic model to characterize its rate and strain-history-dependent behavior. Hence, the total stress response of the liver tissue under combined loading of compression and torsion is expressed as $\boldsymbol{\sigma}^{\mathbf{t}}=\boldsymbol{\sigma}^{\mathbf{e}}+\boldsymbol{\sigma}^{\mathbf{v}}$, where $\boldsymbol{\sigma}^{\mathbf{e}}$ and $\boldsymbol{\sigma}^{\mathbf{v}}$ are the hyperelastic and viscoelastic Cauchy stress tensors (see Fig. 2). In the following sections, we further explain how each stress component is formulated.

\subsubsection{Hyperelasticity}

We assume that the soft tissue is isotropic, homogenous, and incompressible. Using the cylindrical coordinates, a point in the tissue sample is initially located at $\mathbf{X}=\mathbf{X}(\mathrm{R}, \Theta, \mathrm{Z})$ with respect to the reference configuration and at $\mathbf{x}=\mathbf{x}(\mathrm{r}, \theta, \mathrm{z})$ with respect to the deformed configuration.

Then, the deformation gradient for the tissue sample is defined as $\mathbf{F}=\partial \mathbf{x} / \partial \mathbf{X}$. In the case of uniaxial compression of the sample, $r=R / \sqrt{\lambda}, \theta=\Theta, z=\lambda Z$, where, $\lambda$ is the stretch (see 
Fig. 3). Hence, the deformation gradient of a cylindrical tissue sample under pure uniaxial compression is given as

$$
\mathbf{F}_{\mathbf{c}}=\left[\begin{array}{ccc}
1 / \sqrt{\lambda} & 0 & 0 \\
0 & 1 / \sqrt{\lambda} & 0 \\
0 & 0 & \lambda
\end{array}\right]
$$

In the case of simple torsion of the sample, $r=R, \theta=\Theta+K Z, z=Z$, where $K$ is the angle of twist per unit length (see Fig. 3). Then, the deformation gradient of the cylindrical tissue sample under simple torsional shear loading is defined by $\mathbf{F}_{\mathbf{s}}$ as

$$
\mathbf{F}_{\mathbf{s}}=\left[\begin{array}{lll}
1 & 0 & 0 \\
0 & 1 & \gamma \\
0 & 0 & 1
\end{array}\right]
$$

where, $\gamma=\mathrm{rK}$ is the torsional shear strain.

The deformation gradient of an initially compressed cylindrical tissue sample under torsional shear loading is defined by $\mathbf{F}=\mathbf{F}_{\mathbf{s}} \cdot \mathbf{F}_{\mathbf{c}}$ as

$$
\mathbf{F}=\left[\begin{array}{ccc}
\frac{1}{\sqrt{\lambda}} & 0 & 0 \\
0 & \frac{1}{\sqrt{\lambda}} & \gamma \lambda \\
0 & 0 & \lambda
\end{array}\right]
$$

Accordingly, the resulting left Cauchy-Green deformation tensor, $\mathbf{B}=\mathbf{F} . \mathbf{F}^{\mathrm{T}}$, is calculated as

$$
\mathbf{B}=\left[\begin{array}{ccc}
\frac{1}{\lambda} & 0 & 0 \\
0 & \gamma^{2} \lambda^{2}+\frac{1}{\lambda} & \gamma \lambda^{2} \\
0 & \gamma \lambda^{2} & \lambda^{2}
\end{array}\right]
$$

The principle invariants of the left Cauchy-Green tensor are calculated as $\mathrm{I}_{1}=\operatorname{tr}(\mathbf{B})=\gamma^{2} \lambda^{2}+$ $\lambda^{2}+2 / \lambda, \mathrm{I}_{2}=1 / 2\left(\operatorname{tr}(\mathbf{B})^{2}-\operatorname{tr}\left(\mathbf{B}^{2}\right)\right)=\gamma^{2} \lambda^{4}+\gamma^{2} \lambda+2 \lambda+1 / \lambda^{2}, I_{3}=|\mathbf{B}|=1$. 
Using the left Cauchy-Green deformation tensor, the constitutive relation for isotropic, homogenous, and incompressible hyperelastic soft tissue can be written as

$$
\boldsymbol{\sigma}^{\mathrm{e}}=\alpha_{1} \mathbf{B}+\alpha_{2} \mathbf{B}^{2}-\mathrm{p}_{\mathrm{e}} \mathbf{I}
$$

where, $\alpha_{1}=2\left(\frac{\partial \mathrm{W}}{\partial \mathrm{I}_{1}}+\mathrm{I}_{1} \frac{\partial \mathrm{W}}{\partial \mathrm{I}_{2}}\right), \quad \alpha_{2}=-2 \frac{\partial \mathrm{W}}{\partial \mathrm{I}_{2}}$, and $\mathrm{p}_{\mathrm{e}}$ is the undetermined pressure due to the incompressibility condition, and $\mathbf{I}$ is the identity tensor. For a Neo-Hookean material, the strain energy function is defined as $W=\mu / 2\left(I_{1}-3\right)$, where $\mu$ is the linear shear modulus.

\subsubsection{Viscoelasticity}

The constitutive relationship for an isotropic, homogenous, and incompressible viscoelastic soft tissue can be defined as (Pouriayevali et al., 2012; Shim et al., 2004; Yang et al., 2000)

$$
\boldsymbol{\sigma}^{\mathrm{v}}=\mathbf{F}(\mathrm{t}) \cdot \boldsymbol{\Omega}\{\mathbf{C}(\tau)\} \cdot \mathbf{F}^{\mathrm{T}}(\mathrm{t})-\mathrm{p}_{\mathrm{v}} \mathbf{I}
$$

where, $\boldsymbol{\Omega}$ is a matrix functional describing the effect of strain history on stress, $\mathbf{C}$ is the right Cauchy-Green deformation tensor defined as $\mathbf{F}^{\mathrm{T}}$. $\mathbf{F}, \mathrm{p}_{\mathrm{v}}$ is the undetermined pressure due to the incompressibility condition, and $t$ is the time. Based on the constitutive model shown in Fig. 2, the function $\boldsymbol{\Omega}$ takes the following form (Yang et al., 2000)

$$
\boldsymbol{\Omega}\{\mathbf{C}(\tau)\}=\int_{0}^{t} \beta\left(I_{1}, I_{2}\right) m(t-\tau) \dot{E}(\tau) d \tau
$$

where, the Green strain tensor is defined by $\mathbf{E}=(\mathbf{C}-\mathbf{I}) / 2, \beta$ is a function defined as $\beta=A+B\left(I_{2}^{c}-3\right)$. The material parameters $A$ and $B$ represent the linear and nonlinear tangent moduli of the spring in each Maxwell arm shown in Fig. 2, respectively (Please note that the second invariant $I_{2}^{c}=\frac{1}{2}\left(\operatorname{tr}(\mathbf{C})^{2}-\operatorname{tr}\left(\mathbf{C}^{2}\right)\right)$ is related to the deviatoric aspects of the stress and strain). For infinitesimal deformation, $\mathrm{I}_{2}^{\mathrm{c}}-3$ is approximately zero and hence, the proposed viscoelastic model reduces to the well-known generalized Maxwell model. The function $\mathrm{m}(\mathrm{t})$ represents the sum of series of relaxation functions defined for each Maxwell arm as 


$$
m(t-\tau)=\sum_{i=1}^{N} e^{-(t-\tau) / \rho_{i}}
$$

where, $\rho$ is the relaxation time and $\mathrm{N}$ is the number of Maxwell arms connected in parallel. In our earlier studies, we observed that $\mathrm{N}=2$ is sufficient for characterizing the viscoelastic response of liver tissue under compression (Samur et al., 2007). With the same notion, we utilize two additional Maxwell arms for modeling the viscoelastic response of the liver tissue under torsional shear loading. Hence, the rate-dependent viscoelastic behavior of the liver tissue in combined loading of compression and torsional shear is characterized by a total of four Maxwell arms as

$$
\begin{aligned}
& \boldsymbol{\Omega}\{\mathbf{C}(\tau)\}=\int_{0}^{\mathrm{t}_{\mathrm{c}}} \sum_{\mathrm{i}=1}^{2}\left[\mathrm{~A}_{\mathrm{c}, \mathrm{i}}+\mathrm{B}_{\mathrm{c}, \mathrm{i}}\left(\mathrm{I}_{2}^{\mathrm{c}}-3\right)\right] \mathrm{e}^{-\left(\mathrm{t}_{\mathrm{c}}-\tau\right) / \rho_{c, i}} \dot{\mathbf{E}}_{\mathbf{c}} \mathrm{d} \tau \\
& +\int_{0}^{\mathrm{t}_{\mathrm{s}}} \sum_{\mathrm{i}=1}^{2}\left[\mathrm{~A}_{\mathrm{s}, \mathrm{i}}+\mathrm{B}_{\mathrm{s}, \mathrm{i}}\left(\mathrm{I}_{2}^{\mathrm{c}}-3\right)\right] \mathrm{e}^{-\left(\mathrm{t}_{\mathrm{s}}-\tau\right) / \rho_{s, \mathrm{i}}} \dot{\mathbf{E}}_{\mathbf{s}} \mathrm{d} \tau
\end{aligned}
$$

where, the subscripts "c" and "s" represent the variables defined for compression and torsional shear, respectively. The strain rate functions for compression $\left(\dot{\mathbf{E}}_{\mathbf{c}}\right)$ and torsion $\left(\dot{\mathbf{E}}_{\mathbf{s}}\right)$ are given as

$$
\dot{\mathbf{E}}_{\mathbf{c}}=\left[\begin{array}{ccc}
-\frac{\dot{\lambda}}{2 \lambda^{2}} & 0 & 0 \\
0 & -\frac{\dot{\lambda}}{2 \lambda^{2}} & \frac{\gamma \dot{\lambda}}{4 \sqrt{\lambda}} \\
0 & \frac{\gamma \dot{\lambda}}{4 \sqrt{\lambda}} & \frac{1}{2}\left(2 \lambda \dot{\lambda} \gamma^{2}+2 \lambda \dot{\lambda}\right)
\end{array}\right] \quad \dot{\mathbf{E}}_{\mathbf{s}}=\left[\begin{array}{ccc}
0 & 0 & 0 \\
0 & 0 & \frac{1}{2} \sqrt{\lambda} \dot{\gamma} \\
0 & \frac{1}{2} \sqrt{\lambda} \dot{\gamma} & \gamma \lambda^{2} \dot{\gamma}
\end{array}\right]
$$

where, $\dot{\lambda}$ and $\dot{\gamma}$ are the stretch (or strain) and shear rates, respectively. To evaluate the compression data, we set $\gamma=0, \dot{\gamma}=0$ (hence, the second integral term drops automatically) while the superposition principle is utilized (i.e. both terms of the integral are used) to evaluate the combined loading data.

\subsubsection{Hyper-Viscoelasticity}

The total stress response of the liver tissue under combined loading of compression and torsion is given as 


$$
\sigma^{\mathrm{t}}=\sigma^{\mathbf{e}}+\boldsymbol{\sigma}^{\mathbf{v}}
$$

The total hydrostatic pressure, $\mathrm{p}=\mathrm{p}_{\mathrm{e}}+\mathrm{p}_{\mathrm{v}}$, is determined by means of the equilibrium equation, $\operatorname{div} \boldsymbol{\sigma}^{\mathbf{t}}=0$, and using traction free boundary conditions as suggested by Ciarletta and Destrade (2014). Finally, the normal force (NF) during compression and the torque (T) during torsion can be calculated using the components of the stress tensor as:

$$
\begin{aligned}
& N F=2 \pi \int_{0}^{R} r \sigma_{33}^{t} d r \\
& T=2 \pi \int_{0}^{R} \sigma_{23}^{t} r^{2} d r
\end{aligned}
$$

\section{Results}

In order to determine the optimum material parameters of the proposed hyper-viscoelastic model, the experimental data obtained from the normal compression and the dynamic shear loading experiments are used simultaneously along with an optimization scheme utilizing the least-square approach (see Fig. 4). Hence, the goal of the optimization is to estimate the material coefficients of the model $\left(\mu, A_{c, 1}, A_{c, 2}, A_{s, 1}, A_{s, 2}, B_{c, 1}, B_{c, 2}, B_{s, 1}, B_{s, 2}, \rho_{c, 1}, \rho_{c, 2}, \rho_{s, 1}, \rho_{s, 2}\right)$ by minimizing the error between the experimental data and the corresponding values generated by the model. The material parameters estimated by the optimization process are tabulated in Table 2.

The normal force versus strain response of the bovine liver for different loading rates is given in Fig. 5. The markers and error bars represent the mean experimental data (average of 3 animals) and the standard deviations from the mean values, respectively. The solid curves show the corresponding model output $\left(\mathrm{R}^{2}>0.98\right)$. As shown in the figure, the stress response is highly nonlinear, which becomes more prominent with the loading rate.

The average torque response of the liver samples as a function of frequency for compressive pre-strains of $\varepsilon=1 \%$, 2.5\%, 5\%, 7.5\%, 10\%, 12.5\%, 15\%, 17.5\% and 20\%, applied 
at compression rate of $\mathrm{v}=0.000625 \mathrm{~mm} / \mathrm{s}$, is shown in Fig. 6. The markers and solid curves represent the mean experimental data and model output $\left(\mathrm{R}^{2}>0.91\right)$, respectively. As shown in the figure, the magnitude of torque response increases with the frequency and the compressive pre-strain. This plot clearly shows the significant effect of compression on the shear response of the soft tissue. Also, using the torque response at frequencies $\omega=0.1 \mathrm{~Hz}, 1 \mathrm{~Hz}$ and $10 \mathrm{~Hz}$ and the physical dimensions of the tissue samples, the mean (average of 3 animals) values for storage and loss (shear) moduli, are calculated for different compressive pre-strains (Table 3). At low frequency $\left(\omega=0.1 \mathrm{~Hz}\right.$ ), the storage and loss moduli of the samples vary between $\mathrm{G}_{\mathrm{S}}=306-945$ $\mathrm{Pa}$ and $\mathrm{G}_{\mathrm{L}}=56-150 \mathrm{~Pa}$, respectively. When the frequency of stimulation is increased to $10 \mathrm{~Hz}$, the storage and loss moduli of the samples vary between $\mathrm{G}_{\mathrm{S}}=658-2148 \mathrm{~Pa}$ and $\mathrm{G}_{\mathrm{L}}=60-363 \mathrm{~Pa}$, respectively. The results show that the storage and loss shear moduli of bovine liver increase as a function of frequency and compressive pre-strain.

Finally, the "true" dynamic shear response of bovine liver for zero pre-compression $(\varepsilon=$ 0 ) is extrapolated by the proposed hyper-viscoelastic model fitted to the experimental data. Hence, the "true" response comes from a hypothetical measurement, which is not possible to perform in real world since some amount of compression is always necessary for rheological shear measurements. With respect to the true response, the mean relative error (MRE) in the dynamic shear response of bovine liver samples, measured at different compressive pre-strains, is calculated and plotted in Fig. 7 and also tabulated in Table 4. For this purpose, each curve shown in Fig. 6 is subtracted from the true dynamic shear response and the average relative error is reported as MRE. The figure shows that there is a cubic relation between the MRE and the compressive pre-strain. The statistical differences between the dynamic shear responses of bovine liver measured at different compressive pre-strains and the true dynamic shear response corresponding to zero compressive strain are investigated by paired t-tests (Bonferroni correction is applied). The dynamic shear moduli of bovine liver measured at compressive pre-strains higher than $\varepsilon=2.5 \%$ is found to be statistically different than its true shear moduli estimated for zero compressive strain $(\mathrm{p}<0.05)$. 


\section{Discussion}

The compression experiments show the rate-dependent hyperelastic behavior of liver tissue. The proposed hyper-viscoelastic model fit well to the experimental force-strain curves obtained for different compression rates. If the small strain assumption is used, the linear elastic modulus of the bovine liver can be estimated as $\mathrm{E}=3 \mu=801 \mathrm{~Pa}$ (assuming that the liver tissue is homogenous, isotropic, and incompressible). This value is in agreement with linear elastic modulus estimated for bovine liver (between $\mathrm{E}=1600-33600 \mathrm{~Pa}$ as a function of preservation period varying from 5 to $53 \mathrm{~h}$ ) in Yarpuzlu et al. (2014), (as E $5000 \mathrm{~Pa}$ for the preservation period of 1-4 h) in Ocal et al. (2010), (between $E=400-700$ Pa using an ultrasound device and between $\mathrm{E}=300-1600 \mathrm{~Pa}$ using a mechanical tensile testing device) in Chen et al. (1996), shear modulus estimated for bovine liver $(\mathrm{G}=600 \mathrm{~Pa})$ in Liu and Bilston (2000), $(\mathrm{G}=3400 \mathrm{~Pa})$ in Sapin-de Brosses et al. (2010), linear elastic modulus for pig liver ( $~ 10000 \mathrm{~Pa}$ ) in Ottensmeyer (2001), Kruse et al. (2000), Tay et al. (2006), Samur et al. (2007) and elastic modulus for human liver (E $20000 \mathrm{~Pa}$ ) in Nava et al. (2008).

The results of the dynamic shear loading experiments show that the shear modulus of bovine liver increases with frequency, compressive pre-strain, and the rate of compression. We observed that the storage and loss shear moduli of bovine liver, measured at temperature of $24^{\circ} \mathrm{C}$ and torsional shear strain of $\gamma=0.5 \%$, vary between $\mathrm{G}_{\mathrm{S}}=306-2148 \mathrm{~Pa}$, and $\mathrm{G}_{\mathrm{L}}=56-363 \mathrm{~Pa}$ respectively for frequency of stimulation varying between $\omega=0.1-10 \mathrm{~Hz}$ and the compressive pre-strain varying between $\varepsilon=1-20 \%$. These results are compatible with those of the earlier studies. Liu and Bilston (2000) measured the shear storage and loss moduli of the bovine liver under $\varepsilon=5 \%$ compressive pre-strain at $37^{\circ} \mathrm{C}$ temperature to vary between $\mathrm{G}_{\mathrm{S}}=1000-7000 \mathrm{~Pa}$ and $\mathrm{G}_{\mathrm{L}}=300-1000 \mathrm{~Pa}$, respectively for the torsional shear strain of $\gamma=0.11 \%$ and the frequency range of $\omega=0.006-20 \mathrm{~Hz}$. Valtorta and Mazza (2005) used a torsional resonator to characterize the complex shear modulus of bovine liver under $0.2 \mathrm{~Pa}$ vacuum pressure for the frequency range of $\omega=1-10 \mathrm{kHz}$ at the ambient temperature. The results of the experiments suggested that the magnitude of the complex shear modulus of the liver varies between $\left|\mathrm{G}^{*}\right|=5000-20000 \mathrm{~Pa}$. Wex et al. (2013) performed strain ( $\omega=1 \mathrm{~Hz}, \gamma=0.0001-1 \%)$, stress ( $\omega=1 \mathrm{~Hz}, \tau=0.1-100 \mathrm{~Pa}$ ), and frequency sweeps $(\omega=0.1-10 \mathrm{~Hz}, \gamma=0.001 \%)$ and relaxation experiments $(\omega=1 \mathrm{~Hz}, \tau=1 \mathrm{~Pa}$, 
for $600 \mathrm{~s}$ ) on porcine liver using a rheometer under a constant pre-load of $0.6 \mathrm{~N}$. The magnitude of the complex shear modulus was reported to vary between $\left|G^{*}\right|=564-1004 \mathrm{~Pa}$ and $\left|\mathrm{G}^{*}\right|=470$ $578 \mathrm{~Pa}$ under frequency sweep for the post-mortem period of $1 \mathrm{~h}$ and 20-27 h, respectively. Ayyildiz et al. (2014) performed amplitude ( $\omega=20 \mathrm{~Hz}, \gamma=0.1-2 \%$ ) and frequency sweep tests $(\omega=0.1-20 \mathrm{~Hz}, \gamma=1 \%)$ on bovine liver tissue under $\varepsilon=5 \%$ pre-strain and investigated its dynamic shear response as a function of preservation solution and period. The LVR of the bovine liver samples was determined as $\gamma_{\mathrm{LVR}}=1 \%$. The storage modulus of the samples stored in Ringer, HTK and UW solutions varied between $\mathrm{G}_{\mathrm{S}}=361-2084 \mathrm{~Pa}, \mathrm{G}_{\mathrm{S}}=262-701 \mathrm{~Pa}$ and $\mathrm{G}_{\mathrm{S}}=$ 266-2073 Pa, respectively. Also, the loss modulus of the samples stored in Ringer, HTK and UW solutions measured as $\mathrm{G}_{\mathrm{L}}=78-675 \mathrm{~Pa}, \mathrm{G}_{\mathrm{L}}=51-187 \mathrm{~Pa}$, and $\mathrm{G}_{\mathrm{L}}=64-815 \mathrm{~Pa}$, respectively. Klatt et al. (2010) conducted rheological experiments with bovine liver samples, at torsional shear strain of $\gamma=0.3 \%$ and temperature of $1^{\circ} \mathrm{C}$, in the frequency range of $\omega=2.5-62.5 \mathrm{~Hz}$. The storage and loss moduli were varied between $\mathrm{G}_{\mathrm{S}}=1000-3000 \mathrm{~Pa}$ and $\mathrm{G}_{\mathrm{L}}=400-1270 \mathrm{~Pa}$, respectively. Kruse et al. (2000) measured the magnitude of the complex shear modulus of the porcine liver at $37^{\circ} \mathrm{C}$ as $\left|\mathrm{G}^{*}\right|=3000 \mathrm{~Pa}$ at $\omega=100 \mathrm{~Hz}$ and as $\left|\mathrm{G}^{*}\right|=5000 \mathrm{~Pa}$ at $\omega=300 \mathrm{~Hz}$ by using the MRE method. Sapin-de Brosses et al. (2010) measured the magnitude of complex shear modulus of bovine liver as $\left|G^{*}\right|=3400 \pm 500$ Pa by using supersonic shear imaging technique for the frequency range of $\omega=150-200 \mathrm{~Hz}$ at a temperature of $25^{\circ} \mathrm{C}$ and for post-mortem period of 0-48 h. Riek et al. (2011) used MRE technique to examine the dynamic mechanical properties of bovine liver tissue at a temperature of $17-19^{\circ} \mathrm{C}$ for the frequency range of $\omega=100-800 \mathrm{~Hz}$ and post-mortem period of $0-2 \mathrm{~h}$. The authors reported the storage and loss moduli of bovine liver tissue as $\mathrm{G}_{\mathrm{S}}=1420-4910 \mathrm{~Pa}$ and $\mathrm{G}_{\mathrm{L}}=740-1620 \mathrm{~Pa}$, respectively.

The dynamic elastic moduli of animal and human livers have been also investigated in the literature. Ocal et al. (2010) measured the elastic storage and loss moduli of the bovine liver at the room temperature as $E_{S}=5000-80000 \mathrm{~Pa}$ and $E_{L}=1000-13000 \mathrm{~Pa}$, respectively for the frequency range of $\omega=0-80 \mathrm{~Hz}$ and post-mortem period of 1-48 h. Kiss et al. (2004) applied cyclic stimuli $(\omega=0.1-400 \mathrm{~Hz})$ to the canine liver tissue in the normal direction within $72 \mathrm{~h}$ of post-mortem time at $21^{\circ} \mathrm{C}$ and estimated the storage and loss modulus as $\mathrm{E}_{\mathrm{S}}=4000-10000 \mathrm{~Pa}$ and $E_{L}=800-10000 \mathrm{~Pa}$, respectively. Ocal et al. (2010) performed impact hammer experiments on 
fresh human livers harvested from the patients having some form of liver disease and observed that the storage moduli of the livers having no fibrosis (F0) and that of the cirrhotic livers (F4) varied from $E_{S}=10000-20000 \mathrm{~Pa}$ and $E_{L}=20000-50000 \mathrm{~Pa}$ for the frequency range of $\omega=0-80$ $\mathrm{Hz}$, respectively.

The differences in the measurement devices, measurement methods, and the measured subjects (human, porcine, bovine, canine) contribute significantly to the discrepancies in the results of the studies reported above. Another reason for the discrepancy could be the effect of measurement conditions. The environmental conditions such as temperature and humidity affect the material properties of the soft tissue. The storage and loss moduli of the liver tissue were shown to reduce with increasing temperatures (Geerligs et al. (2008); Kiss et al. (2009); Klatt et al. (2010). Nicolle and Palierne (2010) reported that dehydration of tissue samples leads to overestimation of shear modulus. Similarly, post-mortem time, sample preservation method and solution are also significant factors affecting the outcomes of the measurements. Garo et al. (2007) and Ayyildiz et al. (2014) showed a significant increase in dynamic shear moduli of brain and liver tissues for post-mortem interval longer than $6 \mathrm{~h}$ and $11 \mathrm{~h}$, respectively. Ayyildiz et al. (2014) demonstrated that the type of chemical solution used for the preservation of the tissue samples has an influence on the estimation of dynamic shear moduli. In addition, the sample preparation process also has an impact on the measured material properties. Hollenstein et al. (2006); Umale et al. (2011) reported that the liver tissue feels softer when the Glisson's capsule is removed during the sample preparation. Hrapko et al. (2008) showed that obtaining tissue samples from different cutting planes cause variations in the shear material properties due to the anisotropy. There are also inherent difficulties in material characterization of soft tissues using a rheometer, which affect the outcome of the measurements. For example, to prevent slippage between the plates of the rheometer and sample, a sand paper is typically attached to the plates or the sample is glued to the plates. Nicolle and Palierne (2010) compare the use of sandpaper versus adhesive in rheological measurements and conclude that both methods have drawbacks.

There are limited numbers of studies in the literature investigating the effect of compressive pre-strain and strain rate on the measurement of dynamic material properties of liver tissue. Our results showed that the mean relative error in the measurement of dynamic shear 
modulus of bovine liver varied between $\mathrm{MRE}=0.2-243.1 \%$ for the compressive pre-strain varying between $\varepsilon=1-20 \%$. Hrapko et al. (2008) used a rotational rheometer to perform dynamic frequency tests to investigate the effect of compression on shear response. The authors showed that the magnitude of the complex shear modulus of brain tissue is $11.6-21.5 \%$ higher than those measured without pre-compression $(\omega=1-10 \mathrm{~Hz})$ for the compression forces varying between 5-10 mN. Tan et al. (2013) investigated the effect of pre-strain on the viscoelastic behavior of the liver tissue for 2-6 h post mortem time. The authors conducted amplitude sweep experiments at a frequency of $\omega=1 \mathrm{~Hz}$ and torsional shear strain varying between $\gamma=0.005-2 \%$. The storage and loss moduli of liver tissue were reported to vary between $\mathrm{G}_{\mathrm{S}}=350-450 \mathrm{~Pa}$ and $\mathrm{G}_{\mathrm{L}}=70-90$ Pa respectively for the pre compressive strain varying between $\varepsilon=1-20 \%$. DeWall et al. (2012) used dynamic compression testing in normal direction to quantify the viscoelastic properties of 16 human liver samples for a frequency range of $\omega=1-30 \mathrm{~Hz}$ under a compressive strain varying from $\varepsilon=1$ to $6 \%$ (initial compressive pre-strain was $\varepsilon=2 \%$ ) and for a post-mortem period of 0-2 h. The elastic storage and loss moduli of the healthy human liver were observed to increase with compressive strain and varied between $E_{S}=2000-6000 \mathrm{~Pa}$ and $E_{L}=500-2000 \mathrm{~Pa}$, respectively. Nicolle et al. (2010) performed rheological experiments on porcine liver samples at $37^{\circ} \mathrm{C}$ in the frequency range of $\omega=0.1-4 \mathrm{~Hz}$ under strain rates of $0.0151,0.133$, and $0.7 \mathrm{~s}^{-1}$ and for the post-mortem period of 0-24 h. The storage and loss moduli of the samples were measured as $\mathrm{G}_{\mathrm{S}}=800-1100 \mathrm{~Pa}$ and $\mathrm{G}_{\mathrm{L}}=150-300 \mathrm{~Pa}$, respectively.

\section{Conclusion}

In order to perform reliable rheological measurements, some amount of compressive prestrain should be applied on the sample to prevent slippage and maximize grip between the sample and the plates of a rheometer. This work investigates the effect of normal pre-compression on the characterization of the shear material properties of bovine liver tissue. The methodology proposed in this study applies not only to rheological characterization of soft tissues, but also characterization of other viscoelastic solids such as rubber and cheese. Though the effect of compressive pre-strain on the dynamic shear moduli of viscoelastic materials during rheological measurements has been already acknowledged in a few studies in the literature, no systematic 
experimental study, supported by a constitutive model, has been conducted to fully investigate this effect in depth. In this regard, our study is the first to look into this research problem in the domain of soft tissue mechanics, but the same problem also applies to rheological characterization of viscoelastic solids in other domains such as food, automotive, and construction industries.

As reported in this study, the pre-compression has a significant effect on the dynamic shear modulus of soft tissue measured by a rheometer. Our study shows that dynamic shear moduli of bovine liver measured at compressive pre-strains higher than $\varepsilon>2.5 \%$ are statistically different than its true shear moduli, estimated by the model for zero compressive strain ( $\mathrm{p}<$ 0.05). In fact, the results suggest that a mean relative error reaches to $243.1 \%$ in the measurement of dynamic shear moduli of bovine liver under the compressive pre-strain of $\varepsilon=20 \%$ ( $\omega=1-10$ $\mathrm{Hz}$ ). Therefore, for liver tissue, we suggest applying compressive pre-strains not higher than $2.5 \%$ in order to avoid over-estimation of shear material properties. If it is not possible to collect rheological data at lower pre-strains due to contact problems and slippage, then we recommend applying a correction to the measured vales using the relation given in Fig. 7.

\section{Acknowledgements}

The Scientific and Technological Research Council of Turkey (TUBITAK) supported this work under contract MAG-110M649 and the student fellowship program BIDEB-2211.

\section{References}

Ayyildiz, M., Aktas, R.G., Basdogan, C., 2014. Effect of solution and post-mortem time on mechanical and histological properties of liver during cold preservation. Biorheology 51, 47-70.

Chen, E.J., Novakofski, J., Jenkins, W.K., OBrien, W.D., 1996. Young's modulus measurements of soft tissues with application to elasticity imaging. IEEE Transactions on Ultrasonics Ferroelectrics and Frequency Control 43, 191-194.

Ciarletta, P., Destrade, M., 2014. Torsion instability of soft solid cylinders. Ima Journal of Applied Mathematics 79, 804-819.

DeWall, R.J., Bharat, S., Varghese, T., Hanson, M.E., Agni, R.M., Kliewer, M.A., 2012. Characterizing the compression-dependent viscoelastic properties of human hepatic 
pathologies using dynamic compression testing. Physics in Medicine and Biology 57, 2273-2286.

Garo, A., Hrapko, M., van Dommelen, J.A., Peters, G.W., 2007. Towards a reliable characterisation of the mechanical behaviour of brain tissue: The effects of post-mortem time and sample preparation. Biorheology 44, 51-58.

Geerligs, M., Peters, G.W.M., Ackermans, P.A.J., Oomens, C.W.J., Baaijens, F.P.T., 2008. Linear viscoelastic behavior of subcutaneous adipose tissue. Biorheology 45, 677-688.

Hollenstein, M., Nava, A., Valtorta, D., Snedeker, J.G., Mazza, E., 2006. Mechanical characterization of the liver capsule and parenchyma. Biomedical Simulation, Proceedings 4072, 150-158.

Hrapko, M., van Dommelen, J.A., Peters, G.W., Wismans, J.S., 2008. The influence of test conditions on characterization of the mechanical properties of brain tissue. J Biomech Eng 130, 031003.

Kiss, M.Z., Daniels, M.J., Varghese, T., 2009. Investigation of temperature-dependent viscoelastic properties of thermal lesions in ex vivo animal liver tissue. Journal of Biomechanics 42, 959-966.

Kiss, M.Z., Varghese, T., Hall, T.J., 2004. Viscoelastic characterization of in vitro canine tissue. Phys Med Biol 49, 4207-4218.

Klatt, D., Friedrich, C., Korth, Y., Vogt, R., Braun, J., Sack, I., 2010. Viscoelastic properties of liver measured by oscillatory rheometry and multifrequency magnetic resonance elastography. Biorheology 47, 133-141.

Kruse, S.A., Smith, J.A., Lawrence, A.J., Dresner, M.A., Manduca, A., Greenleaf, J.F., Ehman, R.L., 2000. Tissue characterization using magnetic resonance elastography: Preliminary results. Phys Med Biol 45, 1579-1590.

Liu, Z., Bilston, L., 2000. On the viscoelastic character of liver tissue: Experiments and modelling of the linear behaviour. Biorheology 37, 191-201.

Nasseri, S., Bilston, L.E., Phan-Thien, N., 2002. Viscoelastic properties of pig kidney in shear, experimental results and modelling. Rheologica Acta 41, 180-192.

Nava, A., Mazza, E., Furrer, M., Villiger, P., Reinhart, W.H., 2008. In vivo mechanical characterization of human liver. Med Image Anal 12, 203-216.

Nicolle, S., Palierne, J.F., 2010. Dehydration effect on the mechanical behaviour of biological soft tissues: Observations on kidney tissues. Journal of the Mechanical Behavior of Biomedical Materials 3, 630-635.

Nicolle, S., Vezin, P., Palierne, J.F., 2010. A strain-hardening bi-power law for the nonlinear behaviour of biological soft tissues. Journal of Biomechanics 43, 927-932.

Ocal, S., Ozcan, M.U., Basdogan, I., Basdogan, C., 2010. Effect of preservation period on the viscoelastic material properties of soft tissues with implications for liver transplantation. $\mathrm{J}$ Biomech Eng 132, 101007.

Ottensmeyer, M.P., 2001. Minimally invasive instrument for in vivo measurement of solid organ mechanical impedance, Department of Mechanical Engineering. MIT.

Pouriayevali, H., Guo, Y.B., Shim, V.P.W., 2012. A constitutive description of elastomer behaviour at high strain rates - a strain-dependent relaxation time approach. International Journal of Impact Engineering 47, 71-78. 
Riek, K., Klatt, D., Nuzha, H., Mueller, S., Neumann, U., Sack, I., Braun, J., 2011. Wide-range dynamic magnetic resonance elastography. Journal of Biomechanics 44, 1380-1386.

Samur, E., Sedef, M., Basdogan, C., Avtan, L., Duzgun, O., 2007. A robotic indenter for minimally invasive measurement and characterization of soft tissue response. Medical Image Analysis 11, 361-373.

Sapin-de Brosses, E., Gennisson, J.L., Pernot, M., Fink, M., Tanter, M., 2010. Temperature dependence of the shear modulus of soft tissues assessed by ultrasound. Physics in Medicine and Biology 55, 1701-1718.

Shim, V.P.W., Yang, L.M., Lim, C.T., Law, P.H., 2004. A visco-hyperelastic constitutive model to characterize both tensile and compressive behavior of rubber. Journal of Applied Polymer Science 92, 523-531.

Tan, K., Cheng, S., Juge, L., Bilston, L.E., 2013. Characterising soft tissues under large amplitude oscillatory shear and combined loading. Journal of Biomechanics 46, 10601066.

Tay, B.K., Kim, J., Srinivasan, M.A., 2006. In vivo mechanical behavior of intra-abdominal organs. IEEE Trans Biomed Eng 53, 2129-2138.

Umale, S., Chatelin, S., Bourdet, N., Deck, C., Diana, M., Dhumane, P., Soler, L., Marescaux, J., Willinger, R., 2011. Experimental in vitro mechanical characterization of porcine glisson's capsule and hepatic veins. J Biomech 44, 1678-1683.

Valtorta, D., Mazza, E., 2005. Dynamic measurement of soft tissue viscoelastic properties with a torsional resonator device. Medical Image Analysis 9, 481-490.

Wex, C., Stoll, A., Fröhlich, M., Arndt, S., Lippert, H., 2013. How preservation time changes the linear viscoelastic properties of porcine liver. Biorheology 50, 115-131.

Yang, L.M., Shim, V.P.W., Lim, C.T., 2000. A visco-hyperelastic approach to modelling the constitutive behaviour of rubber. International Journal of Impact Engineering 24, 545560.

Yarpuzlu, B., Ayyildiz, M., Tok, O.E., Aktas, R.G., Basdogan, C., 2014. Correlation between the mechanical and histological properties of liver tissue. Journal of the Mechanical Behavior of Biomedical Materials 29, 403-416. 


\section{Figures:}

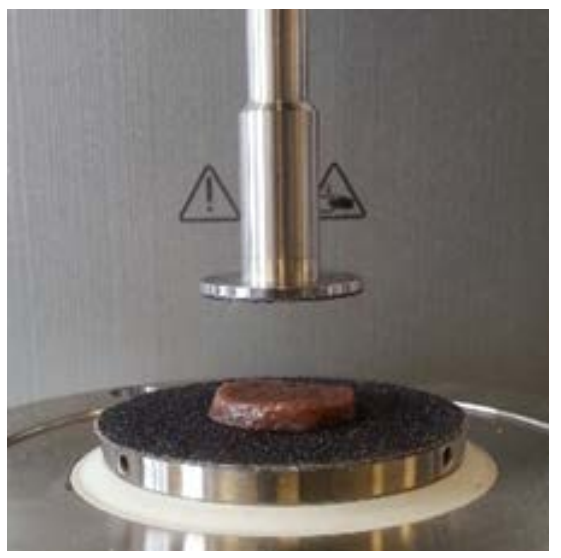

Fig. 1 - A liver tissue sample on the lower plate of the rheometer.

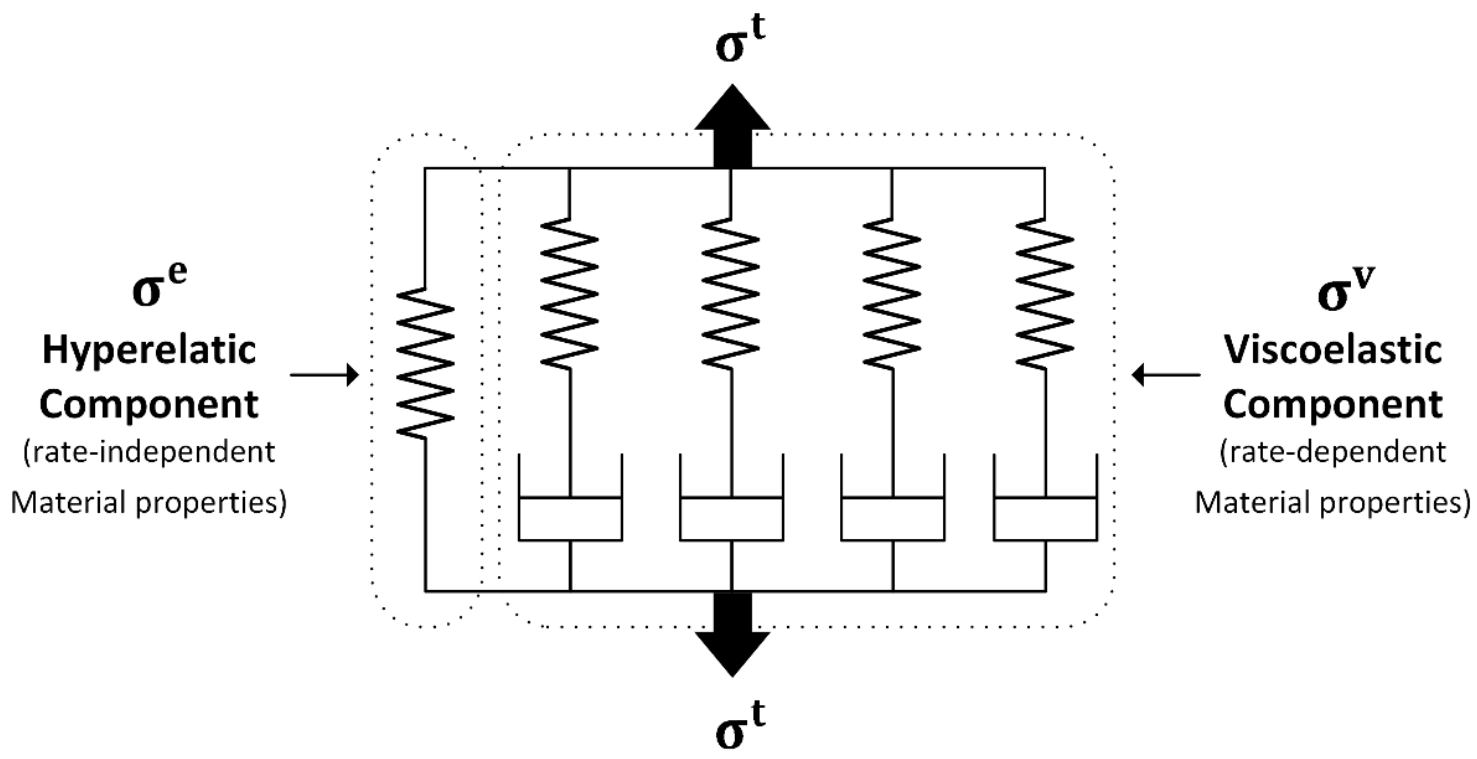

Fig. 2 - The proposed hyper-viscoelastic model of soft tissue. 


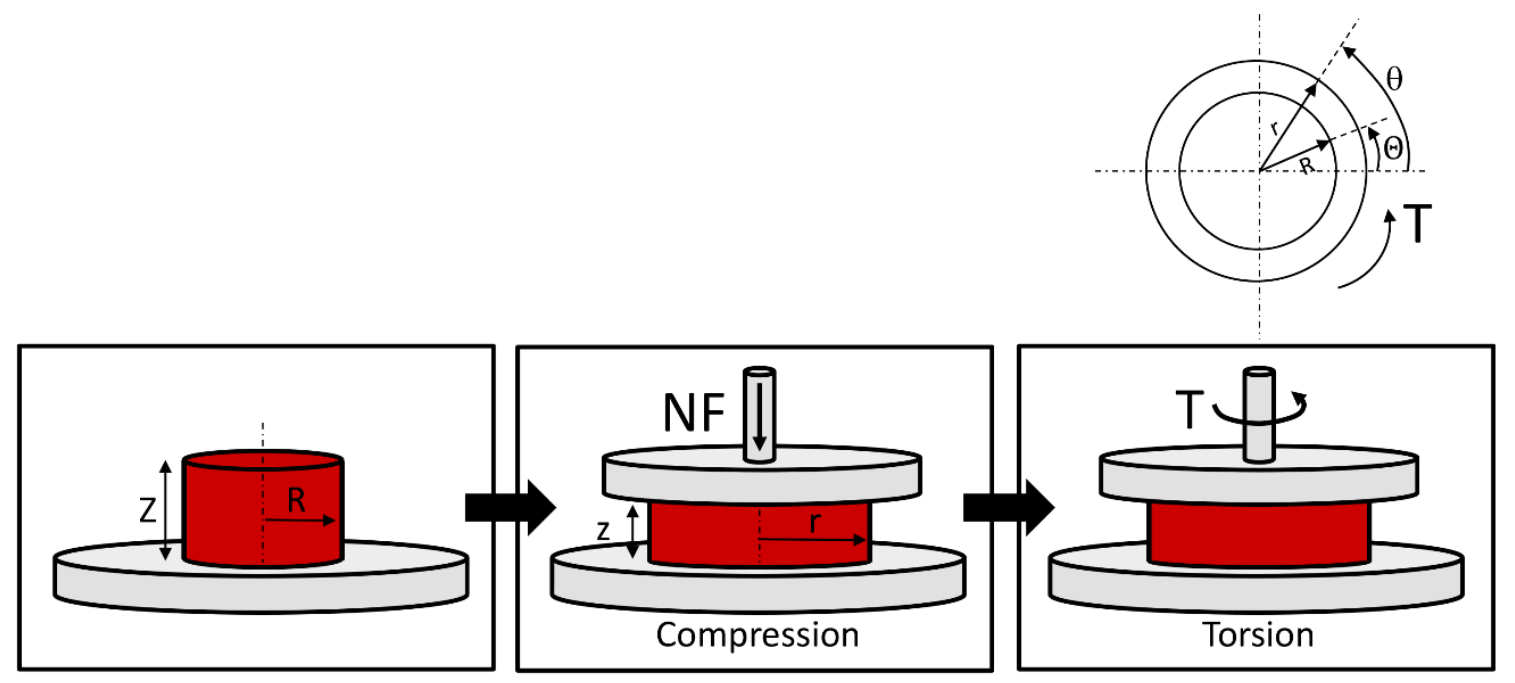

Fig. 3 - The tissue sample in reference configuration is compressed first and then torsion is applied. In the figure, NF and $\mathrm{T}$ represent the normal force and torque, respectively.

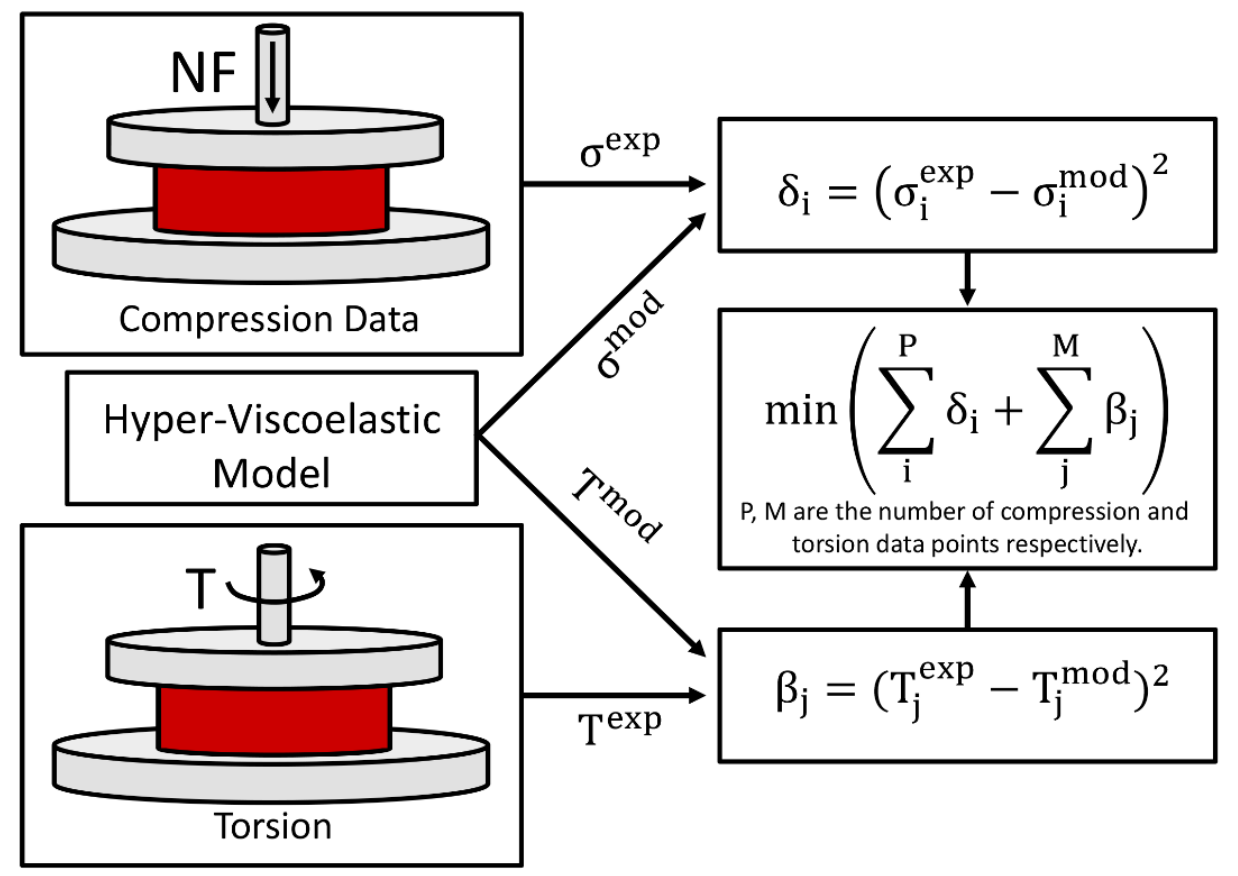

Fig. 4 - The optimization scheme for the estimation of the material parameters of the hyperviscoelastic model. 


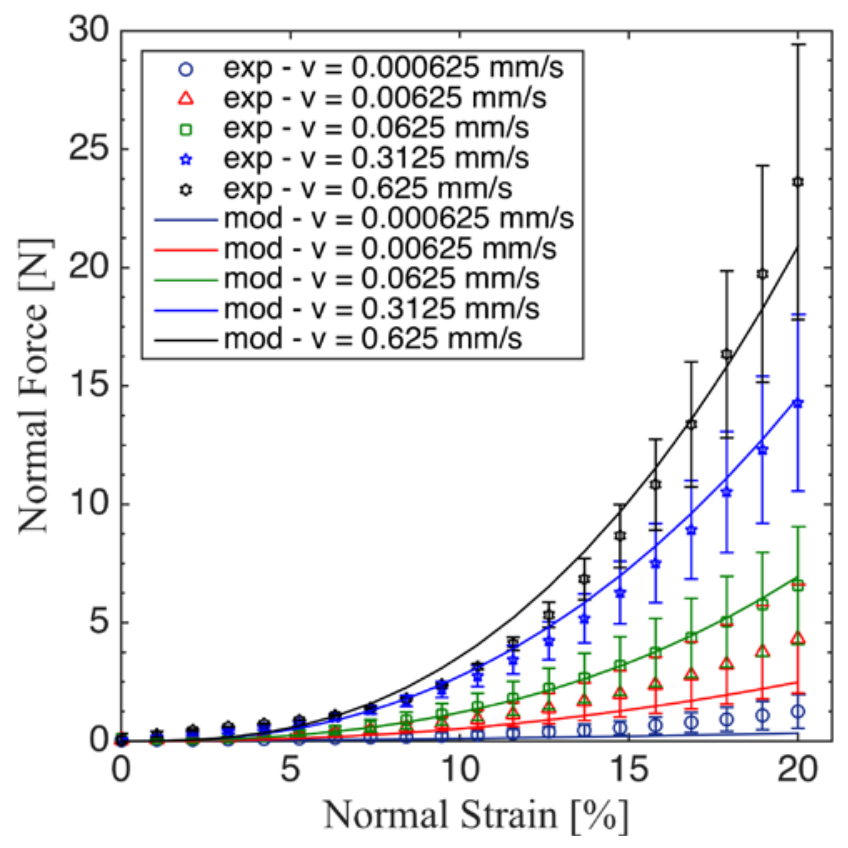

Fig. 5 - The normal force-strain response of bovine liver for different compression rates. The markers and the solid curves represent the mean experimental data and the model output, respectively.
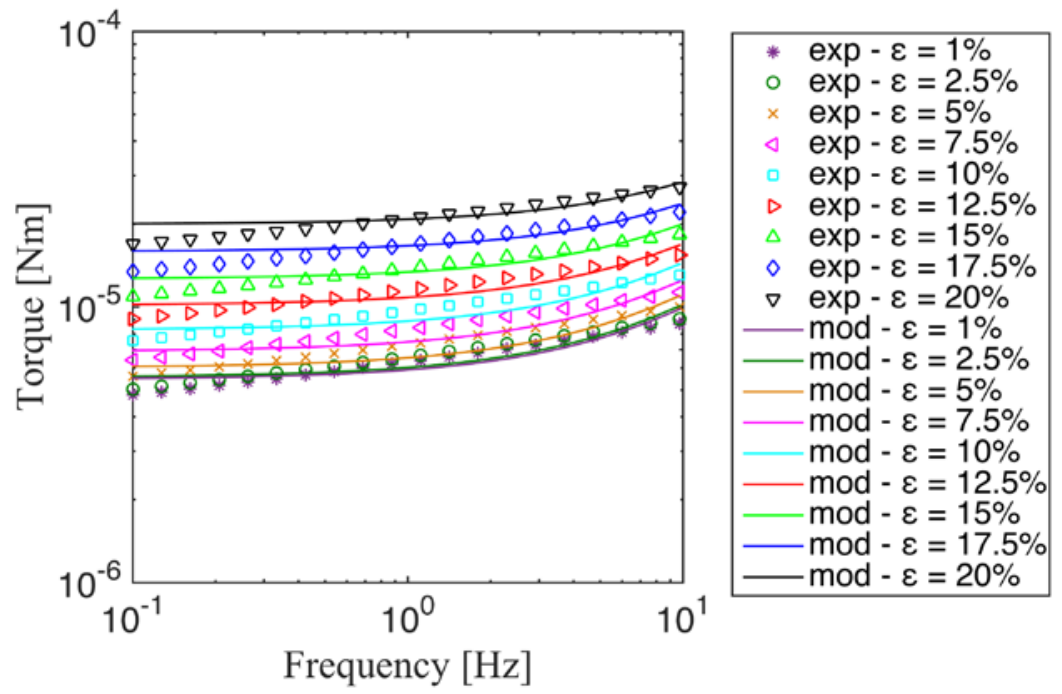

Fig. 6 - The torque response of bovine liver for different compressive pre-strains applied at quasi-static rate of $v=0.000625 \mathrm{~mm} / \mathrm{s}$. The markers and the solid curves represent the mean experimental data and the model output, respectively. 


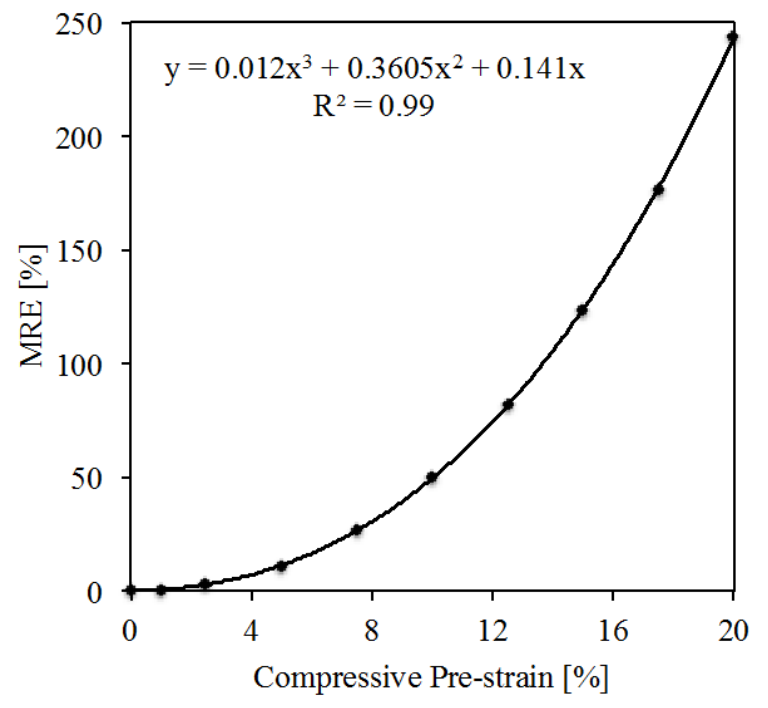

Fig. 7 - The mean relative error (MRE) made in the measurement of dynamic shear modulus of bovine liver as a function of compressive pre-strain. 


\section{Tables:}

Table 1 - Summary of the experiments

\begin{tabular}{|c|c|c|c|c|c|c|}
\hline $\begin{array}{c}\text { Type of } \\
\text { Experiment }\end{array}$ & $\begin{array}{l}\text { Number } \\
\text { of Livers }\end{array}$ & $\begin{array}{c}\text { Number of } \\
\text { Samples/Liver }\end{array}$ & $\begin{array}{c}\text { Compressive } \\
\text { Strain [\%] }\end{array}$ & $\begin{array}{c}\text { Compressive } \\
\text { Strain Rate } \\
{[\mathrm{mm} / \mathrm{s}]}\end{array}$ & $\begin{array}{c}\text { Shear } \\
\text { Strain [\%] }\end{array}$ & $\begin{array}{c}\text { Frequency } \\
{[\mathrm{Hz}]}\end{array}$ \\
\hline \multirow{5}{*}{ Compression } & \multirow{5}{*}{3} & 4 & 20 & 0.000625 & & \\
\hline & & 4 & 20 & 0.00625 & & \\
\hline & & 4 & 20 & 0.0625 & & \\
\hline & & 4 & 20 & 0.315 & & \\
\hline & & 4 & 20 & 0.625 & & \\
\hline Dynamic & \multirow[b]{2}{*}{3} & $\begin{array}{c}4 \\
\text { (for amplitude } \\
\text { sweep) }\end{array}$ & $5,10,15,20$ & 0.000625 & $0.1-5$ & 10 \\
\hline Shear Loading & & $\begin{array}{c}4 \\
\text { (for frequency } \\
\text { sweep) }\end{array}$ & $\begin{array}{c}1,2.5,5,7.5,10 \\
12.5,15,17.5,20\end{array}$ & 0.000625 & 0.5 & $0.1-10$ \\
\hline
\end{tabular}

Table 2 - The material parameters of the hyper-viscoelastic model estimated by the optimization.

\begin{tabular}{|c|c|c|c|c|c|c|c|c|c|c|c|c|}
\hline$\mu[\mathrm{Pa}]$ & $\mathbf{A}_{\mathrm{c}, 1}[\mathrm{~Pa}]$ & $\mathbf{A}_{\mathbf{c}, 2}[\mathrm{~Pa}]$ & $\mathbf{A}_{\mathrm{s}, \mathbf{1}}[\mathrm{Pa}]$ & $\mathbf{A}_{\mathbf{s}, 2}[\mathrm{~Pa}]$ & $\mathrm{B}_{\mathrm{c}, \mathbf{1}}[\mathrm{Pa}]$ & $\mathbf{B}_{\mathrm{c}, 2}[\mathrm{~Pa}]$ & $B_{s, 1}[\mathrm{~Pa}]$ & $\mathrm{B}_{\mathrm{s}, 2}[\mathrm{~Pa}]$ & $\rho_{c, 1}[s]$ & $\rho_{\mathrm{c}, 2}[\mathrm{~s}]$ & $\rho_{\mathrm{s}, 1}[\mathrm{~s}]$ & $\rho_{s, 2}[s]$ \\
\hline 2.67E2 & 1.53E-8 & $8.78 \mathrm{E}-8$ & 2.00E-3 & 8.74E1 & 2.09E6 & 3.61E5 & 1.56E1 & $9.16 \mathrm{E} 1$ & $5.01 \mathrm{E} 1$ & 3.92E1 & $8.28 \mathrm{E} 5$ & $7.85 \mathrm{E}-2$ \\
\hline
\end{tabular}

Table 3 - The storage and loss (shear) moduli of bovine liver measured at $0.1 \mathrm{~Hz}, 1 \mathrm{~Hz}$ and $10 \mathrm{~Hz}$ for different compressive pre-strains.

\begin{tabular}{ccccccc}
\hline \multirow{2}{*}{$\begin{array}{c}\text { Compressive } \\
\text { Pre-strain }(\varepsilon)\end{array}$} & $\begin{array}{c}\text { Storage } \\
\text { Modulus [Pa] }\end{array}$ & $\begin{array}{c}\text { Loss Modulus } \\
{[\mathrm{Pa}]}\end{array}$ & $\begin{array}{c}\text { Storage } \\
\text { Modulus [Pa] }\end{array}$ & $\begin{array}{c}\text { Loss Modulus } \\
{[\mathrm{Pa}]}\end{array}$ & $\begin{array}{c}\text { Storage } \\
\text { Modulus [Pa] }\end{array}$ & $\begin{array}{c}\text { Loss Modulus } \\
{[\mathrm{Pa}]}\end{array}$ \\
\hline $1 \%$ & $306 \pm 14$ & $56 \pm 5$ & $440 \pm 29$ & $94 \pm 10$ & $658 \pm 61$ & $60 \pm 15$ \\
\hline $2.5 \%$ & $320 \pm 11$ & $57 \pm 4$ & $460 \pm 24$ & $98 \pm 7$ & $678 \pm 30$ & $70 \pm 31$ \\
\hline $5 \%$ & $354 \pm 9$ & $63 \pm 4$ & $513 \pm 22$ & $108 \pm 8$ & $726 \pm 56$ & $80 \pm 4$ \\
\hline $7.5 \%$ & $400 \pm 10$ & $70 \pm 4$ & $584 \pm 20$ & $119 \pm 8$ & $867 \pm 44$ & $119 \pm 21$ \\
\hline $10 \%$ & $460 \pm 9$ & $79 \pm 4$ & $681 \pm 17$ & $135 \pm 7$ & $1047 \pm 36$ & $131 \pm 9$ \\
\hline $12.5 \%$ & $541 \pm 10$ & $91 \pm 4$ & $813 \pm 10$ & $155 \pm 5$ & $1221 \pm 57$ & $173 \pm 19$ \\
\hline $15 \%$ & $639 \pm 27$ & $105 \pm 5$ & $980 \pm 30$ & $180 \pm 6$ & $1411 \pm 63$ & $237 \pm 28$ \\
\hline $17.5 \%$ & $768 \pm 36$ & $125 \pm 6$ & $1195 \pm 37$ & $211 \pm 6$ & $1729 \pm 83$ & $296 \pm 22$ \\
\hline $20 \%$ & $945 \pm 60$ & $150 \pm 5$ & $1497 \pm 82$ & $251 \pm 8$ & $2148 \pm 167$ & $363 \pm 7$ \\
\hline
\end{tabular}


Table 4 - The mean relative error (MRE) in dynamic shear modulus of bovine liver measured at different compressive pre-strains. The frequency of stimulation varies between 0.1 and $10 \mathrm{~Hz}$.

\begin{tabular}{ccccccccc}
\hline $\mathrm{MRE}_{\varepsilon=1 \%}$ & $\mathrm{MRE}_{\varepsilon=2.5 \%}$ & $\mathrm{MRE}_{\varepsilon=5 \%}$ & $\mathrm{MRE}_{\varepsilon=7.5 \%}$ & $\mathrm{MRE}_{\varepsilon=10 \%}$ & $\mathrm{MRE}_{\varepsilon=12.5 \%}$ & $\mathrm{MRE}_{\varepsilon=15 \%}$ & $\mathrm{MRE}_{\varepsilon=17.5 \%}$ & $\mathrm{MRE}_{\varepsilon=20 \%}$ \\
\hline $0.2 \%$ & $2.3 \%$ & $11.0 \%$ & $26.6 \%$ & $49.9 \%$ & $81.7 \%$ & $123.3 \%$ & $176.4 \%$ & $243.1 \%$ \\
\hline
\end{tabular}

